\title{
Microstructure formation and macroscopic dynamics of ferrofluid emulsion in rotating magnetic field
}

\author{
Anastasia Kolesnikova ${ }^{1}$, Arthur Zakinyan ${ }^{1, *}$, and Yuri Dikansky ${ }^{1}$ \\ ${ }^{1}$ Department of General and Theoretical Physics, North Caucasus Federal University, 1 Pushkin Street, Stavropol 355009, Russia
}

\begin{abstract}
The ferrofluid-in-water emulsion has been produced and studied. The microstructure formation in a flat layer of such emulsion under the action of in-plane rotating magnetic field has been observed. Several microstructure types have been found such as chain-like aggregates, disc-like clusters, branching and space-occupying structures. The time evolution of the formation process has been analysed. The revealed microstructures have been computationally investigated by molecular dynamics simulations. The microstructure dynamics in ferrofluid emulsions manifests itself in macroscopic mechanical effects. We analysed the appearance of macroscopic torque on a ferrofluid emulsion sample in a rotating magnetic field. The value of the torque has been measured as a function of magnetic field strength and frequency.
\end{abstract}

\section{Introduction}

Processes of formation and growth of clusters from individual particles or components attract great interest in the context of investigation of complex heterogeneous systems. The aggregation process constitutes an important field of basic research and applications in various branches of physics and materials sciences. Many efforts have been undertaken to understand the aggregation dynamics of magnetic microparticles dispersed in a fluid (magnetorheological suspension), and subjected to constant and/or varying magnetic fields. The works [1-3] can be cited as a few examples of the research in this direction. The particles under the action of applied magnetic field undergo an induced, complex self-organization process. In the context of the present research the structurization processes under the rotating uniform magnetic field are of particular interest. In our case, the system under study is a coarse ferrofluid-inwater emulsion. The use of ferrofluid emulsion gives some new opportunities in the aggregation process studies such as the ability to produce the samples with different droplet sizes and the ability to produce the inverse systems of nonmagnetic droplets suspended in ferrofluid.

The microstructure formation dynamics in composite material can manifest itself in macroscopic mechanical effects. Thus the rotating clusters forming in a magnetically active suspension under the action of rotating magnetic field can produce a macroscopic torque on a suspension sample. Previously the effects of internal rotations have been studied with regard to the pure ferrofluids [4-7]. It can be expected that the internal rotation effect will be even more pronounced in magnetic suspensions (such as ferrofluid emulsion) than that in a pure ferrofluid.

\section{Microstructure formation processes}

The ferrofluid emulsion under study has been produced by dispersing a kerosene-based ferrofluid with magnetite nanoparticles in distilled water by means of ultrasonic disperser. Sodium dodecyl sulfate has been used as emulsifier. The emulsion with droplets sizes of a few tens of micrometers has been obtained. The microstructure formation in a flat horizontal layer of such emulsions under the action of in-plane rotating magnetic field has been observed and studied by means of an optical microscope. Uniform rotating magnetic field has been produced by two pairs of perpendicular Helmholtz coils.

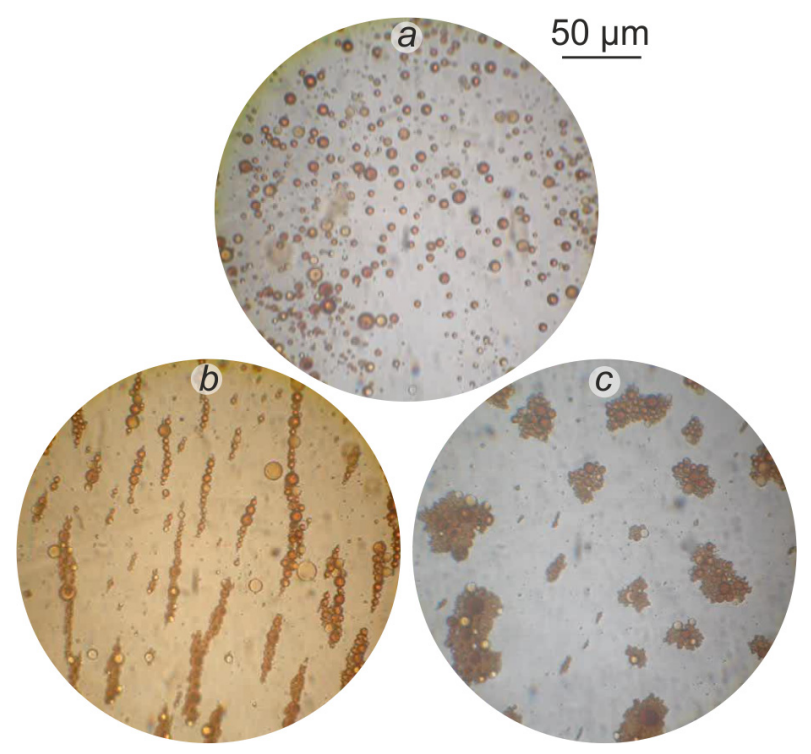

Fig. 1. Microstructures at low concentration. (a) Zero field. (b) Chain microstructure $(f=1 \mathrm{~Hz}, H=9 \mathrm{kA} / \mathrm{m})$. (c) Disc-like microstructure $(f=7 \mathrm{~Hz}, H=7.8 \mathrm{kA} / \mathrm{m})$. 
It has been found several microstructure types. At comparatively low emulsion concentrations two types of clusters have been revealed: chain-like aggregates and disc-like clusters (Fig. 1). At low magnetic field frequencies and high magnetic field strengths the chinelike structures takes place. At higher frequencies and lower magnetic fields the droplet discs are formed. The structures evolve in time and their type can change. Thus the initial chains can transform into discs. The phase diagrams of structure types in frequency-strength coordinates at three time moments are shown in Fig. 2.

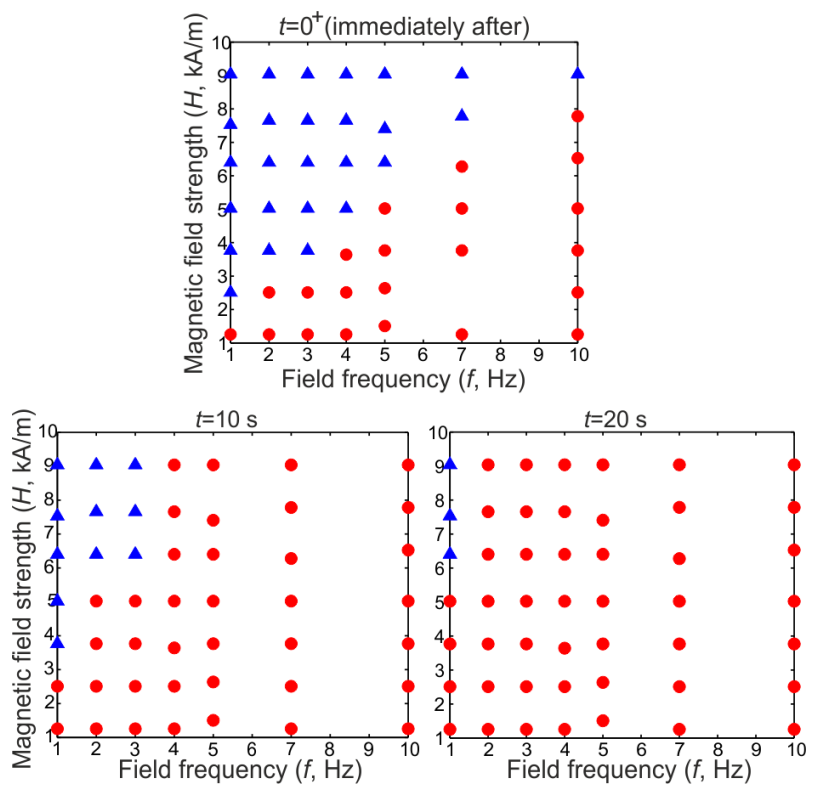

Fig. 2. Phase diagrams of structures at different moments after magnetic field application. Triangles $(\boldsymbol{\Delta})$ correspond to chainlike aggregates; circles $(\bullet)$ correspond to disc-like clusters.

The microstructure time evolution has been analysed be measuring the relative total cluster number. Fig. 3 shows the time dependence of the relative total cluster number at different values of the rotating magnetic field strength. As is seen, this dependence tends to saturation after first ten seconds of magnetic field action. At higher fields the aggregation process is more intensive.

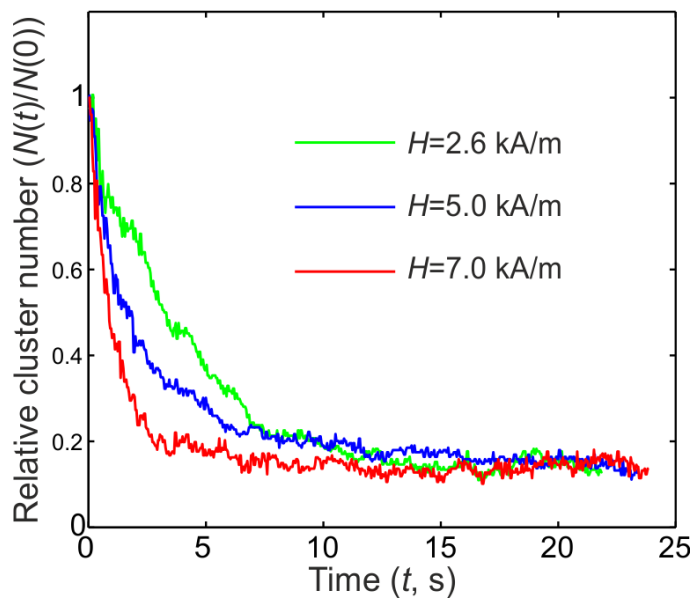

Fig. 3. Time dependencies of the relative cluster number at different values of magnetic field strength. The magnetic field frequency is $f=5 \mathrm{~Hz}$.
The emulsion concentration is also an essential factor for the microstructure formation. It has been found that at comparatively high concentrations the mesoscale structures of particle clusters are formed under the action of rotating magnetic field independently of its frequency and strength. Thus, Fig. 4a shows the branching structures formed at middle concentrations. At higher concentrations the space-occupying structures take place (Fig. 4b).
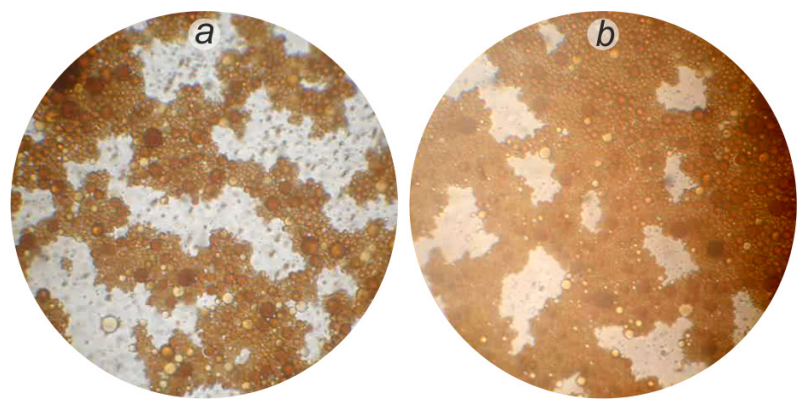

Fig. 4. Branching (a) and space-occupying (b) structures at high concentrations of ferrofluid emulsion.

The studied systems have been computationally investigated by molecular dynamics simulations similar to that presented in [8]. The main interactions that capture the general behavior of the droplets are the dipolar magnetic interaction, the drag force and the repulsive force avoiding droplets from overlapping. In contrast to [8], the local magnetic field was used to calculate the droplets magnetic moments instead of the external field; and Hadamard-Rybczynski formula was used instead of Stokes' formula for the drag force. The structures analogous to the experimentally observed have been obtained in the calculations with the use of experimental values of the system parameters. The results of calculations are shown in Fig. 5.
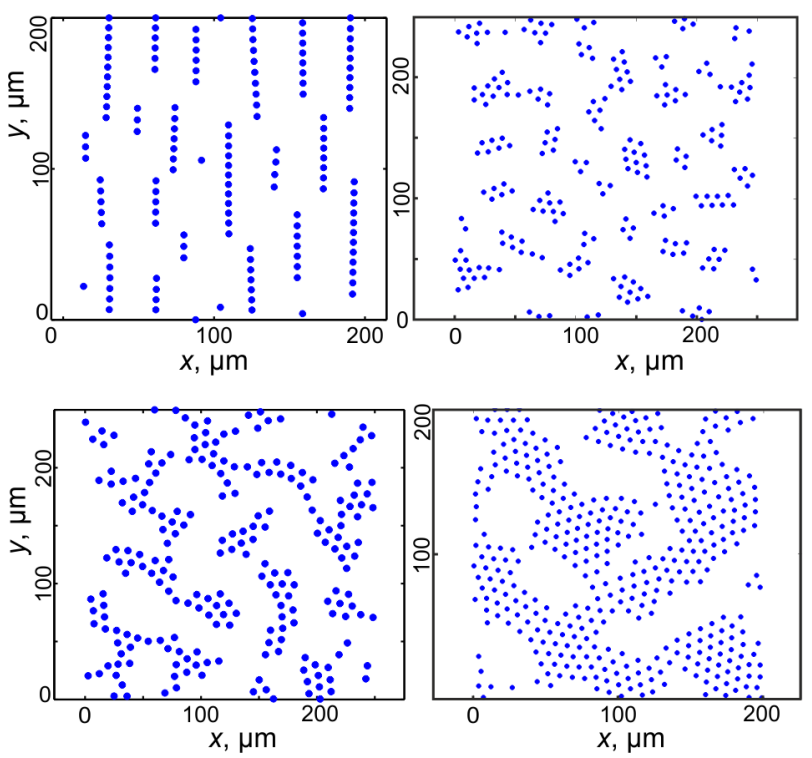

Fig. 5. Results of the molecular dynamic simulations of the microstructure of ferrofluid emulsion under the rotating magnetic field. The chain-like and disc-like aggregates, branching and space-occupying structures can be observed. 
To characterize the emulsion microstructure the pair distribution function has been calculated based on the simulations results (Fig. 6).
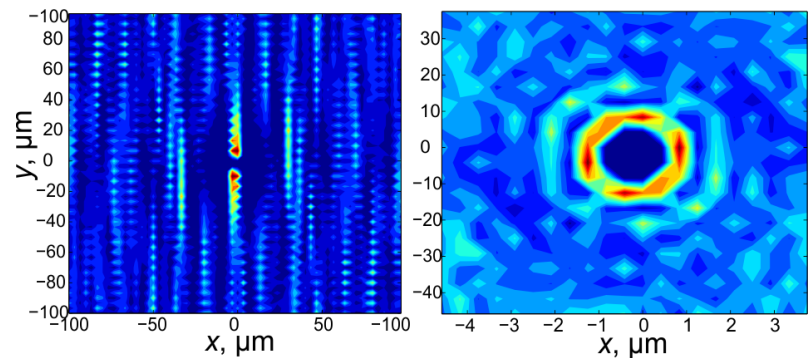

Fig. 6. Pair distribution functions calculated on the basis of simulations results. Left image corresponds to the chain microstructure; right image corresponds to the branching microstructure.

\section{Macroscopic torque measurements}

We have analysed the appearance of macroscopic torque on a ferrofluid emulsion sample in a rotating magnetic field. The internal rotations of formed microstructural elements produce torque acting on a whole emulsion sample due to the viscous coupling. To measure the torque the emulsion sample was placed into a hollow plastic sphere which is neutrally buoyanced in a surrounding liquid. Under the action of uniform rotating magnetic field the sphere rotation has been observed. The sphere rotation frequency has been measured from which the toque acting on sphere can be easily determined. The macroscopic torque generating inside the sphere in a rotating magnetic field due to the structurization processes is balanced by the viscous torque acting on the outside from the surrounding liquid in this case.

The macroscopic torque has been measured as a function of magnetic field strength and frequency. The results of measurements are presented in Fig. 7. As it can be seen, the torque grows with magnetic field strength and frequency.

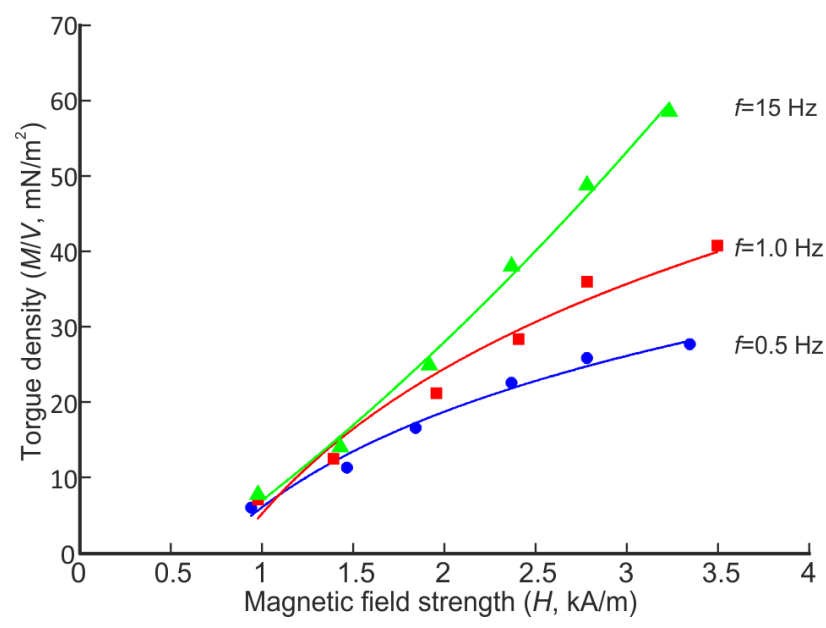

Fig. 7. Macroscopic torque density as a function of magnetic field strength at different values of magnetic field frequency.

\section{Conclusions}

Thus, in the present work the processes of structure formation in a ferrofluid emulsion have been studied experimentally. Different microstructure types have been observed and studied depending on the magnetic field strength, frequency, time and emulsion concentration. The chain-like, disc-like, branching and space-occupying structures have been analyzed. Analogous structures have been obtained in numerical simulations. The appearance of the macroscopic torque in a ferrofluid emulsion due to the internal rotations of formed microstructure elements has been found. It can be concluded that the ferrofluid emulsions can be regarded as controllable soft magnetic materials. The studied effects can be useful for actuation purposes.

This work was supported by the grant of the President of the Russian Federation No. MK-3169.2017.2 and also by the Ministry of Education and Science of the Russian Federation in the framework of the base part of governmental ordering for scientific research works (project № 3.5822.2017/8.9).

\section{References}

1. L.D. Yépez, J.L. Carrillo, F. Donado, J.M. SausedoSolorio, P. Miranda-Romagnoli, J. Magn. Magn. Mater. 408, 321-329 (2016).

2. M. Llera, J. Codnia, G.A. Jorge, J. Magn. Magn. Mater. 384, 93-100 (2015).

3. J. Černák, G. Helgesen, Phys. Rev. E 78, 061401 (2008).

4. M.I. Shliomis, Sov. Phys. JETP 34, 1291-1294 (1972).

5. A.V. Lebedev, A.F. Pshenichnikov, Magnetohydrodynamics 27, 4-8 (1991).

6. A. Chaves, M. Zahn, C. Rinaldi, Phys. Fluids 20, 053102 (2008).

7. I. Torres-Diaz, A. Cortes, Y. Cedeño-Mattei, O. Perales-Perez, C. Rinaldi, Phys. Fluids 26, 012004 (2014).

8. S. Melle, O.G. Calderón, G.G. Fuller, M.A. Rubio, J. Colloid Interf. Sci. 247, 200-209 (2002). 